\title{
Dynamic Behavior of Surface-Enhanced Raman Spectra for Rhodamine 6G Interacting with Gold Nanorods: Implication for Analyses under Wet vs. Dry Conditions
}

Anerise de Barros ${ }^{1 *}$, Flavio Makoto Shimizu ${ }^{2,3}$ Cristine Santos de Oliveira ${ }^{1,4}$, Fernando Aparecido Sigoli ${ }^{1}$, Diego Pereira dos Santos ${ }^{1}$ and Italo Odone Mazali ${ }^{{ }^{*}}$

${ }^{1}$ Laboratory of Functional Materials, Institute of Chemistry, University of Campinas UNICAMP, P.O. Box 6154, 13083-970, Campinas, SP, Brazil.

${ }^{2}$ São Carlos Institute of Physics, University of São Paulo, P.O Box 369, 13560-970, São Carlos, SP, Brazil.

${ }^{3}$ Present address: Brazilian Nanotechnology National Laboratory (LNNano), Brazilian Center for Research in Energy and Materials (CNPEM), 13083-970, Campinas, SP, Brazil.

${ }^{4}$ Present address: Institute of Physics, Martin Luther University Halle-Wittenberg, 06120 Halle (Saale), Germany.

*corresponding author.

E-mail address: mazali@unicamp.br

E-mail address: anerisedebarros@gmail.com 
Fig. S1 corresponds to the schematic illustration of Seed Au nanosphere and the growth Au nanorods procedure. The sequential addition of precursors is described in more detail in the experimental section of the manuscript. The Au seed nanosphere exhibits a light brown color with the transition color to wine brown, as seen in the original colloidal suspension extracted during the synthesis in Fig. S1. Additional images of AuNRs obtained from TEM are illustrated in Fig. S2, and the SERS substrates reproducibility in different days are shown in Fig. S3.
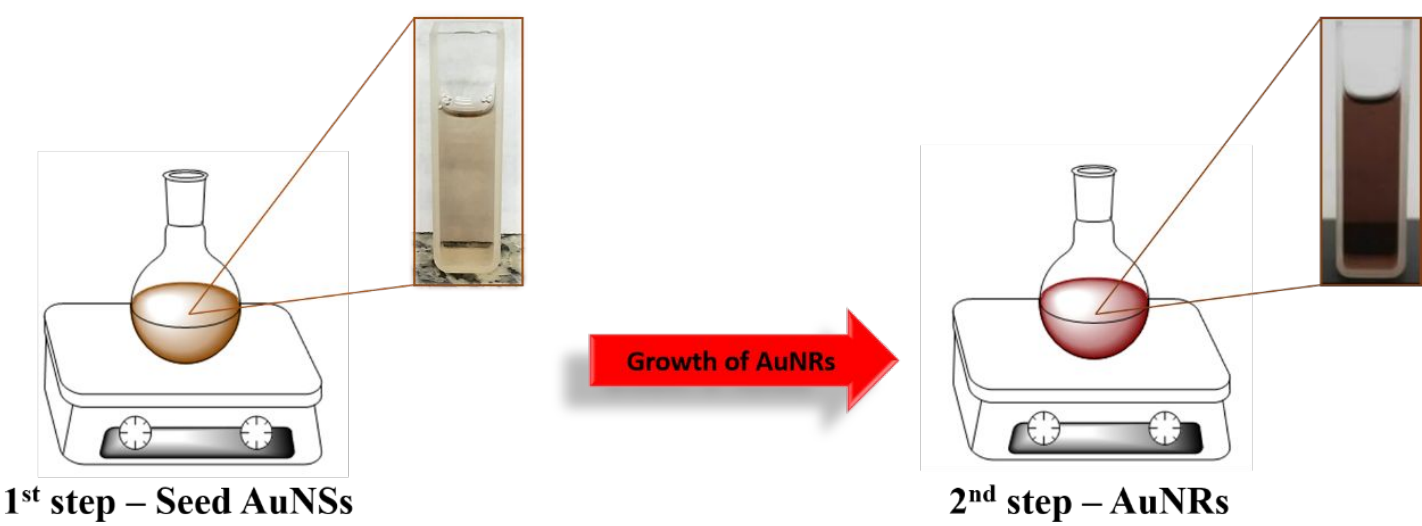

Figure S1. Schematic representation of the synthesis protocol to obtain AuNRs. 


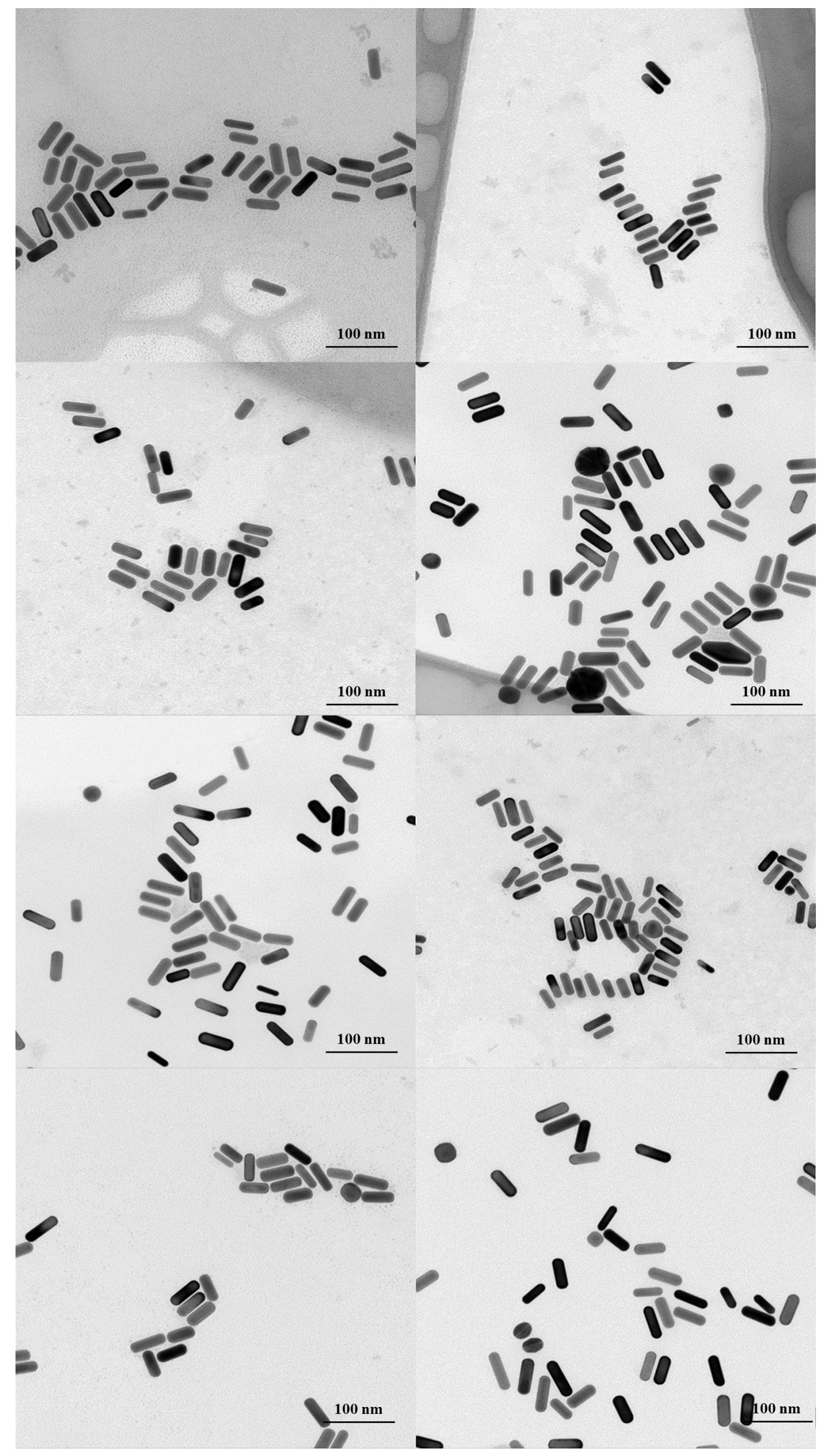

Figure S2. TEM microscopy of AuNRs used for counting size distribution and aspect ratio. 


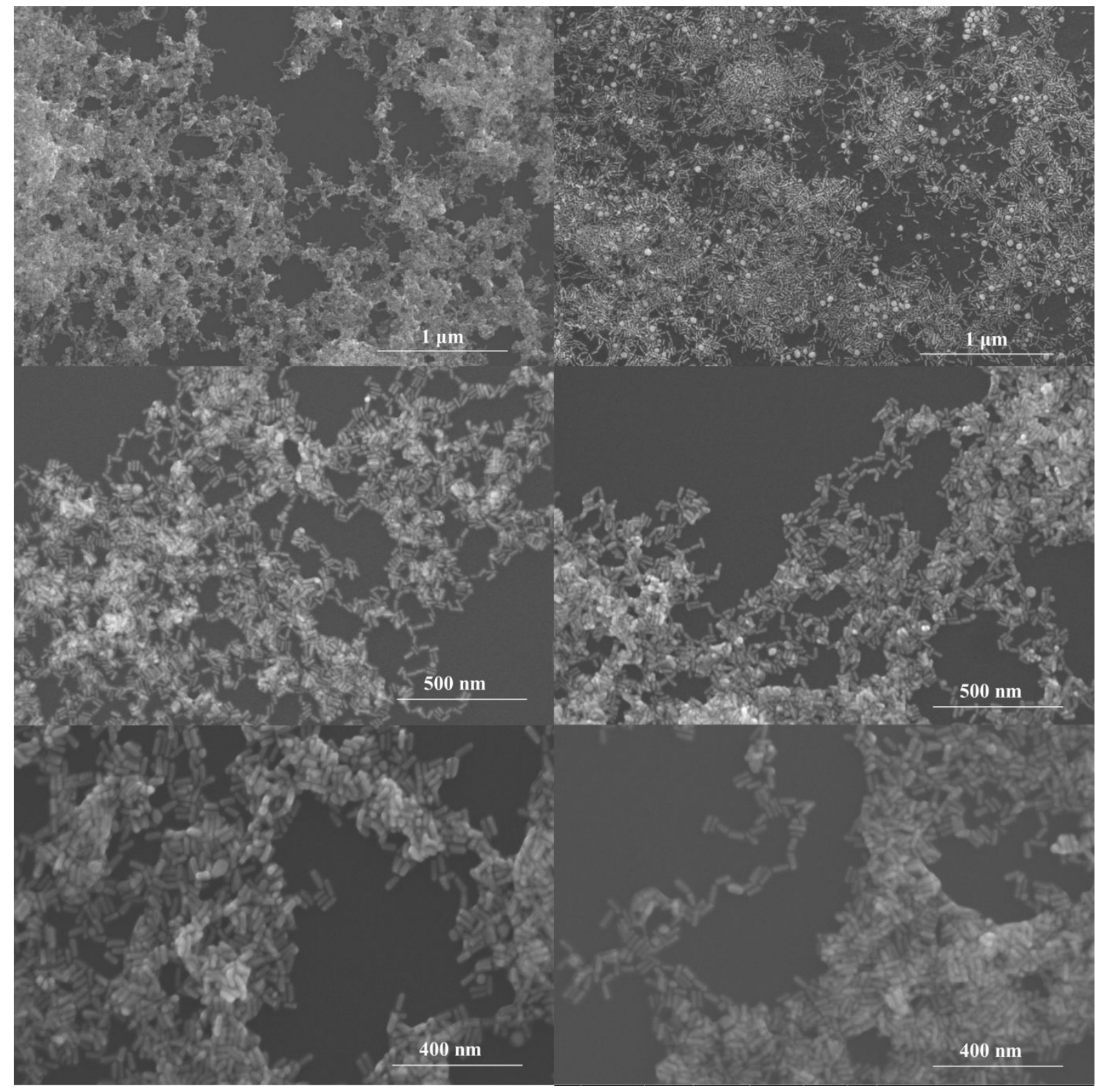

Figure S3. SEM-FEG of SERS substrates obtained by Si functionalization with MPTMS and AuNRs immobilized.

Raman spectrum of R6G aqueous solution on the Si substrate without functionalization with Au nanorods is shown in Fig. S4. The Raman spectrum was collected using an R6G solution in $10^{-2} \mathrm{~mol} \mathrm{~L}^{-1}$ concentration, with an aliquot of $5.0 \mu \mathrm{L}$ dropped on previously cleaned Si substrate. The Raman spectrum was obtained applying $60 \mathrm{~s}$ of time exposition with 1 accumulation, using an objective lens of 50x. A comparison of Raman bands assignment of neat R6G and the bands with SERS spectra in different analysis conditions can be verified in Table S1. Additionally, SERS spectra are shown in Fig. S5 to check the drying process of a drop of water after R6G dried on the SERS substrate, also corroborating the influences in the Raman intensity during the drying process. 


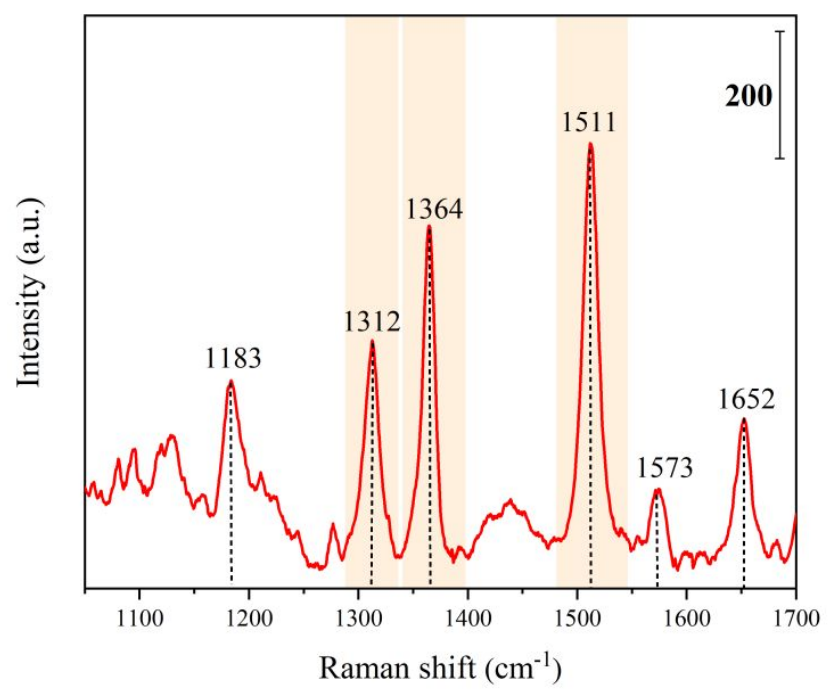

Figure S4. Raman spectrum for dry R6G onto silicon substrate functionalized with MPTMS. The analysis was achieved using $5 \mu \mathrm{L}$ of R6G with a concentration of $10^{-2} \mathrm{~mol} \mathrm{~L}^{-1}$.

Table S1. Assignment of vibrational modes in SERS Spectra of Rhodamine 6G dye.

\begin{tabular}{|c|c|c|c|}
\hline \multicolumn{3}{|c|}{ Raman shift $\left(\mathrm{cm}^{-1}\right)$} & \multirow[t]{2}{*}{ Assignment ${ }^{28,47-49}$} \\
\hline $\begin{array}{c}\text { R6G aqueous solution on } \\
\text { the Si/MPTMS/AuNRs } \\
\text { SERS substrates }\end{array}$ & $\begin{array}{l}\text { R6G dries on the } \\
\text { Si/MPTMS/AuNRs } \\
\text { SERS substrates }\end{array}$ & $\begin{array}{c}\text { Water droplet under } \\
\text { Si/MPTMS/AuNRs SERS } \\
\text { substrates with R6G } \\
\text { dries }\end{array}$ & \\
\hline 1088 & $\begin{array}{l}--- \\
\end{array}$ & $\begin{array}{l}--- \\
\end{array}$ & $\begin{array}{c}\text { Hybrid mode } \\
\text { (xanthene/phenyl rings and } \\
\mathrm{NHC}_{2} \mathrm{H}_{5}\end{array}$ \\
\hline 1126 & 1127 & 1127 & $\begin{array}{l}\mathrm{C}-\mathrm{H} \text { in-plane bending in } \\
\text { xanthene/phenyl rings }\end{array}$ \\
\hline 1182 & 1184 & 1184 & $\begin{array}{c}\mathrm{C}-\mathrm{H} \text { in-plane bending in } \\
\text { xanthene ring }\end{array}$ \\
\hline 1311 & 1311 & 1311 & $\begin{array}{c}\text { Hybrid mode } \\
\text { (xanthene/phenyl rings and } \\
\mathrm{NHC}_{2} \mathrm{H}_{5} \text { group) }\end{array}$ \\
\hline 1361 & 1362 & 1362 & $\begin{array}{c}\mathrm{C}-\mathrm{C} \text { stretching in xanthene } \\
\text { ring }\end{array}$ \\
\hline 1511 & 1508 & 1508 & $\begin{array}{c}\mathrm{C}-\mathrm{C} \text { stretching in xanthene } \\
\text { ring }\end{array}$ \\
\hline 1573 & 1575 & 1575 & $\begin{array}{l}\mathrm{C}-\mathrm{C} \text { stretching in the phenyl } \\
\text { ring }\end{array}$ \\
\hline 1650 & 1648 & 1648 & $\begin{array}{c}\mathrm{C}-\mathrm{C} \text { stretching in xanthene } \\
\text { ring }\end{array}$ \\
\hline
\end{tabular}



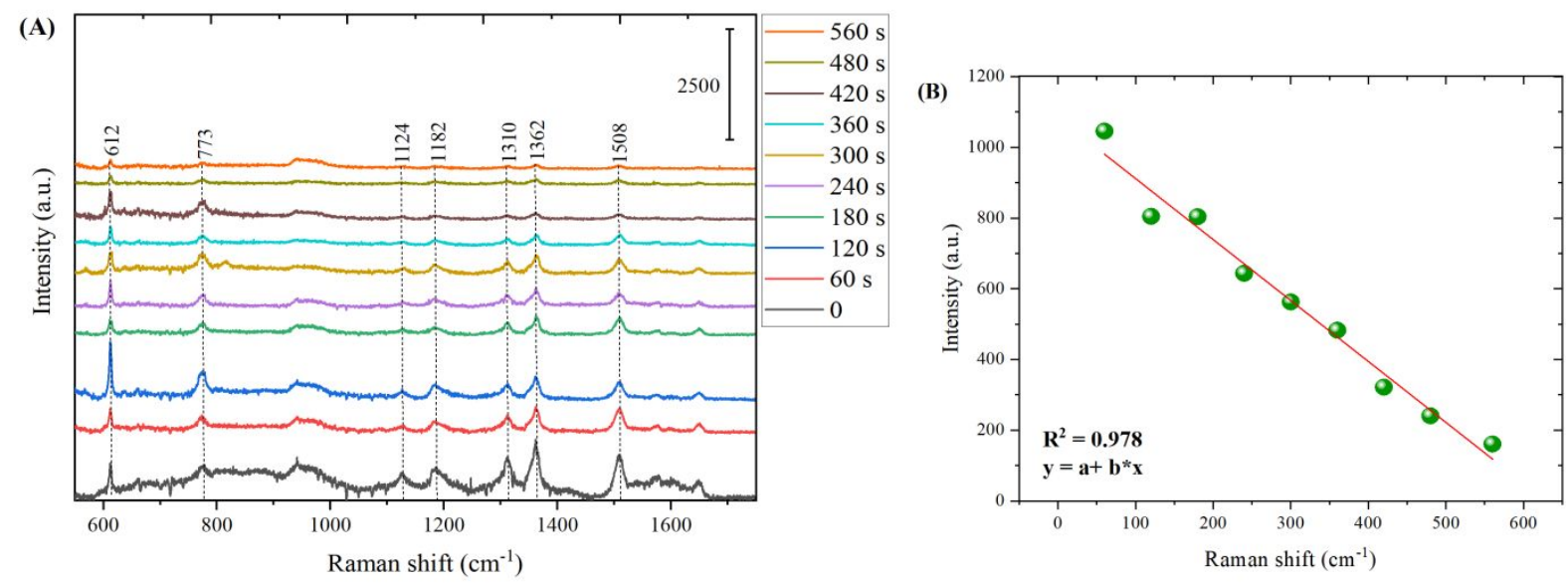

Figure S5. (A) SERS spectra of drying process of drop water on the SERS substrate with R6G already dry. (B) Graphical of Raman intensity $\left(1508 \mathrm{~cm}^{-1}\right)$ as a function of time for a drop of water on the top of SERS substrate with R6G dry. 